\title{
The anti-obesity potential of green tea: The effect on leptin and adiponectin
}

\author{
Kenneth Essex ${ }^{\mathrm{a}}$, Sapha Mosawy ${ }^{* a}$
}

\author{
${ }^{a}$ Menzies Health Institute Queensland, Griffith University, Gold Coast, 4215, Australia
}

\begin{abstract}
Green tea has been consumed for thousands of years, and has been known to have many medicinal properties such as, anti-cancerous, anti-atherogenic, anti-oxidative, anti-inflammatory and anti-bacterial. With the increasing prevalence of cardiovascular disease (CVD), and its major risk factor, obesity, there has been increasing interest in green tea as a potential anti-obesity therapy. Evidence has demonstrated that green tea decreases body weight. However it remains unclear through which pathway and mechanism green tea exerts its anti-obesity properties. The metabolic adipose tissue hormones, adipocytokines, leptin and adiponectin have been studied extensively as a potential target for green tea and its main catechin, epigallacatechin gallate (EGCG). It has been demonstrated that green tea consumption decreases leptin levels which may directly decrease body weight through increasing energy expenditure and fat oxidation. Furthermore, ingestion of green tea or its catechins has been associated with increased adiponectin levels which may indirectly reduce body weight through insulin sensitivity.
\end{abstract}

Keywords: Green tea, leptin, adiponectin, CVD, obesity.

\section{INTRODUCTION}

Tea is considered as one of the most consumed beverages in today's society [1]. It has been consumed for thousands of years, and has been thought to have originated during the Shang dynasty in 1600BC from southwest China [2]. The leaf of the Camellia sinensis plant is the source of tea, with only degrees in fermentation determining the different varieties produced [3]. The most fermented (completely oxidised) variety of tea is black tea, followed by the moderately fermented (partially oxidised) oolong tea. Green tea, non-fermented (non-oxidised) tea, is the closest to its natural pure leaf form, with drying being the only form of processing [3, 4]. Through only drying the raw leaf, green tea is able to retain its natural chemical polyphenols, specifically the polyphenol flavonoid epigallacatechin gallate (EGCG) [4].

EGCG is one of the many flavonoid catechins that make up the chemical components of green tea, with EGCG making up 48-55\% [5]. Green tea and its catechins have been extensively studied in the past few decades, with multiple studies providing evidence of its beneficial effects, specifically focusing on its potential role in reducing the rapidly increasing incidence of cardiovascular disease (CVD) [6]. Large body of research has demonstrated that green tea has anti-inflammatory, anti-oxidative, antithrombotic and lipid lowering properties, all of which play an essential role in decreasing the risk of CVD [7, 8].

\footnotetext{
*Address correspondence to Sapha Mosawy at Menzies Health Institute Queensland. Griffith University, Gold Coast, Australia; Tel/Fax: ++61755527031; E-mails: s.mosawy@griffith.edu.au
}

We have demonstrated the anti-platelet potential of EGCG in vitro [9]. In addition, green tea's potential role in controlling obesity via its effect on the adiopocytokines adiponectin and leptin, both of which play suspected roles in glucose regulation via insulin [8, 10, 11], and energy expenditure respectively [11-14] have been investigated. The present review focuses on the effect of green tea on leptin and adiponectin as potential targets to reduce obesity.

\section{Obesity}

Obesity prevalence has increased significantly in recent times, not only in the developed world but also in the developing world [15] and is rapidly becoming an emerging global public health issue [16]. With the increases in energy dense foods and sedentary lifestyles in today's modern society, obesity has allowed CVD to become the number one cause of death globally [14, 17]. Obesity has been extensively studied and has been implicated in many cardiovascular diseases such as coronary heart disease (CAD), stroke, heart failure and hypertension [16, 18]. Generally, obese individuals consume a diet rich in saturated fats, which are high in cholesterol, and this elevation in cholesterol increases the risk of atherosclerotic plaques which can cause stroke, CAD [19, 20] and myocardial infarctions [16]. In addition, obesity can also lead to high blood pressure, which can cause left ventricular hypertrophy $(\mathrm{LVH})$, type 2 diabetes and increased abdominal and visceral adipose tissue stores $[16,21]$.

Green tea has been demonstrated to reduce body weight, blood pressure and cholesterol [22]. Indeed, in a 90 day randomized controlled trial involving moderately overweight adults ingesting $1,900 \mathrm{mg} /$ day of green tea. It was demonstrated that green tea consumption reduced body weight and waistline. Furthermore, significant weight 
reduction was achieved when obese adults were placed specially prepared diet that included green tea [23, 24].

\section{Adipocytokines}

Adipose tissue has long been thought to have only one main function, the storage of fat. However, it has been shown to play a much larger role in the body's metabolic system. Adipose tissue is as an endocrine organ that releases adipocytokines $[14,25]$. There are several types of adipocytokines involved with varying biological processes ranging from inflammation (tumour-necrosis factor (TNF) and intereukin-6 (IL-6)) [26], angiogenesis (vascular endothelial growth factor (VEGF)) [27] and metabolism (leptin and adiponectin) [11]. Leptin and adiponectin are thought to regulate energy homeostasis via the control of hunger, insulin sensitivity and energy metabolism through increased energy expenditure [28, 29]. These two hormones have been identified as potential targets to reduce obesity.

\subsection{Leptin}

Leptin is one of several adipocytokine hormones released from adipocytes [30]. It is a peptide hormone involved in the regulation of energy homeostasis via satiety and inhibition of hunger [13]. Leptin is synthesised exponentially in adipocytes in response to increasing fat stores [31] and binds to leptin receptors in the arcuate nucleus of the hypothalamus (ARC) [28, 29]. Leptin binds to ObRb, which is the major isoform of the leptin receptor responsible for initiating anorexigenic signalling $[12,13]$. Once bound several signalling pathways are stimulated, including JAKSTAT [32], MAPK, P13K/AKT and AMPK, all of which activate two groups of neurons; cocaine/amphetamineregulated transcript (CART) peptides and proopiomelanocortin (POMC) [12, 33], Fig. (1). Proopiomelanocortin (POMC) releases the anoretic peptide $\alpha$ melanocyte-stimulating hormone ( $\alpha$-MSH) [12], and both of these hormones cause decrease in food consumption and appetite via the activation of satiety, as well as increasing metabolic rate through energy expenditure (catabolism) [28, 29].
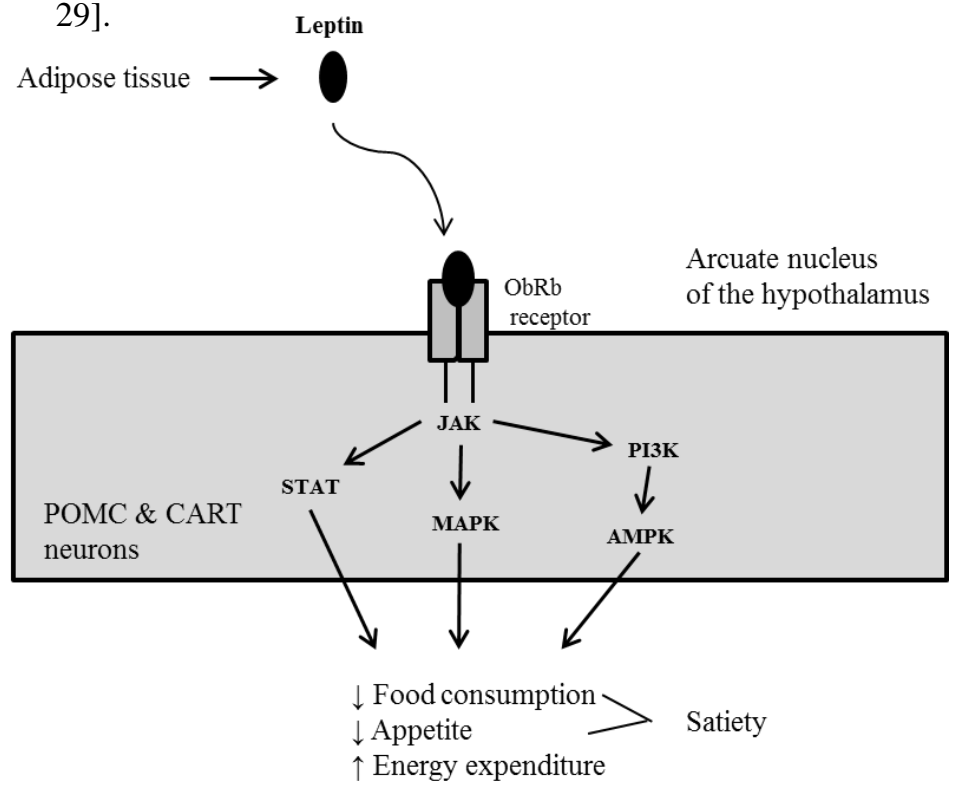

Fig. (1). Schematic representation of leptin pathway and its effects on energy metabolism and obesity.

\subsubsection{Effect of green tea on leptin}

One way of reducing obesity and its associated harmful effects is to utilise naturally occurring substances. Green tea and its constituents have investigated for their anti-obesity potential. Constituents of Green tea have been demonstrated to reduce body weight and serum lipids [34]. In addition, increase in body weight and intraperitoneal adipose tissues were reported to have been significantly reduced following the consumption of diets containing 2 and $4 \%$ green tea powder [35]. It has been postulated that the anti-obesity effects are mediated via increased energy expenditure and lipid oxidation in which leptin plays an important role [30]

Leptin has been identified as a potential target to reduce body fat and control food intake. Previous studies have reported evidence that green tea consumption reduces obesity through lowering leptin levels and its effect on the hypothalamus. Auvichayapat et al. [36] in an in vivo randomised controlled study using green tea involving 60 obese human subjects. In this study leptin, satiety and energy expenditure were investigated. It was demonstrated that over a 12 week period there was a statistically significant difference in weight between both the green tea group and their baseline levels. Leptin levels in the green tea group were lower than the control group. Furthermore, in the green tea group resting energy expenditure recorded higher [36].

Another study by Al-Sowyan [34] reported a reduction in serum leptin levels following a 14 day green tea diet in albino male rats. Furthermore, it was demonstrated that reduced leptin levels were a companied by a reduction in body and adipose weight [34]. Lee et al [37] also demonstrated reduction in body weight post EGCG consumption. In this study 3 groups of mice, 1 group fed without EGCG (control group), and 2 groups fed with varying concentrations of EGCG $(0.2 \%$ and $0.5 \%)$, were fed a high fat diet for a period of 8 weeks. It was reported decreased adipose tissue mass. Gene expression of regulatory element-binding protein-1c (SREBP-1c), adipocyte fatty acid-binding protein (aP2), lipoprotein lipase (LPL) and fatty acid synthase (FAS) was also significantly decreased.

A study by Dulloo et al. [38] conducted over a 24-hour period on 3 separate occasions involving human volunteers consuming green tea extract (equivalent to $270 \mathrm{mg}$ of EGCG). It was demonstrated following EGCG ingestion there was a $4 \%$ increase in energy expenditure. This was mediated by inhibition of catechol $O$-methyltransferase (COMT) [38]. By inhibiting the enzyme COMT, norepinephrine concentrations in synaptic clefts are not degraded, and as a result increase or prolong the effects of the sympathetic nervous system (SNS), a system that largely controls thermogenesis (energy expenditure) and fat oxidation [38]. Leptin has not only been shown to affect satiety, but it also affects energy expenditure, and further evidence demonstrates that leptin decreases COMT levels [39]. However, a recent study by Lorenz et al [40] demonstrated that COMT activity is not inhibited by high doses of EGCG, suggesting a negligible role of COMT in the catechin effects in vivo. The majority of studoes clearly demonstrate that the anti-obesity potential of green tea. 
However, further studies are still required to fully investigate the effect of green tea on leptin production and the antiobesity mechanisms.

Although leptin has shown to reduce body weight in obese individual, there are evidences of leptin resistance. It has been reported that in some obese individuals high levels of levels of leptin failed to reduce weight and suppress hunger [41]. There are several mechanisms have been postulated to explain leptin resistance in obesity. These mechanisms include changes in the transport of leptin across the blood brain barrier and impaired leptin receptor signalling [24]. Therefore, further studies are warranted to investigate the effect of green tea consumption on leptin resistance.

\subsection{Adiponectin}

Adiponectin, first described and characterised in mid 1990 s, is another main adipocytokine peptide hormone that is exclusively synthesised and released from adipocytes [42]. Adiponectin is found circulating in serum in 3 forms; trimer, hexamer and high molecular weight adiponectin (HMW), with the most active form being HMW adiponectin [42]. Adiponectin has been suggested to play an essential role in modulating lipid and glucose metabolism. Decreased levels of adiponectin have been associated with insulin resistance in obesity [35]. Furthermore, a large body of research has concluded that adiponectin levels have a strong correlation in insulin insensitivity in type 2 diabetics [42, 43]. It has been shown that adiponectin exerts multiple effects through its main receptors AdipoR1 and AdipoR2. Adipo R1 is abundantly found in skeletal muscle, while AdipoR2 is found in liver [23], Fig. (2) shows schematic representation of adiponectin effects on energy metabolism. Additional studies have provided evidence that adiponectin has multiple biological effects on homeostasis of the vascular system, in that it is effected by inflammatory mechanisms that are specifically associated with CVD [25]. It has also been postulated that adiponectin could potentially be used as a biomarker for obesity related diseases, and to monitor disease progression, however there has been conflicting evidence on its effective use as a biomarker [25].

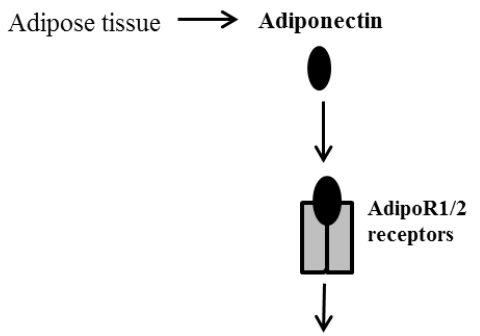

Activation of AMPK \& PAPA a
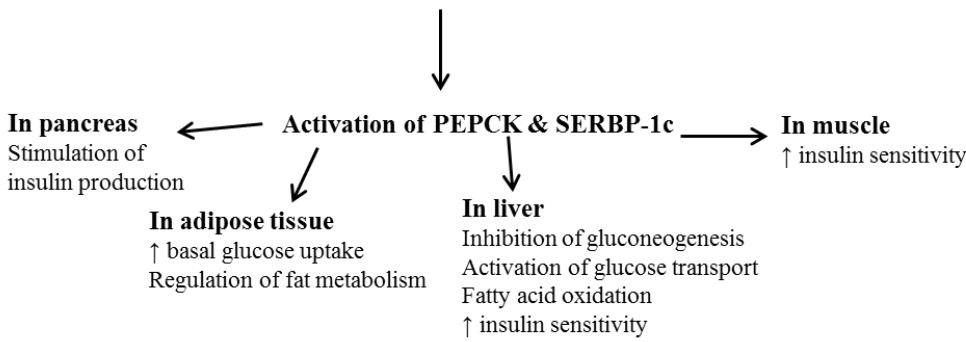

Fig. (2). Adiponectin to its receptors induces activation of AMP kinase (AMPK) and peroxisome proliferator-activated receptor $\alpha(\mathrm{PPAR} \alpha)$ and subsequently the activation of
Phosphoenolpyruvate carboxykinase (PEPCK) and sterol regulatory element-binding protein 1c (SERBP-1c).

\subsubsection{Effect of green tea on adiponectin}

Green tea consumption has been shown to increase adiponectin levels in both human subjects and animal models. An animal study reported by Tian et al. [44] investigating reduction in fat deposition in rats following the administration of green tea polyphenols. Green tea was administrated at three different concentrations $(0.8,1.6$ and $3.2 \mathrm{~g} / \mathrm{L}$ ), for a duration of 26 weeks. In the high fat fed rats it was demonstrated that there was a decreased expression in both peroxisome proliferator-activated receptors (PPAR-y), the receptor that is responsible for adiponectin gene transcription in visceral adipose tissue [8], and serum adiponectin. However, rats that consumed the green tea polyphenols had a decrease in visceral fat stores, while their adiponectin level increased in comparison to the high fat fed rats. Furthermore, green tea administration significantly decreased phosphorylation of PPAR- $y$, while increasing the gene expression of adiponectin. Increased adiponectin levels post green tea consumption was also reported in human subjects. A12-week randomized controlled trial that involved consumption of $458 \mathrm{mg} / \mathrm{day}$ of green tea catechins by obese adults showed increase in adiponectin levels. In addition, there was significant decrease in cholesterol, triglycerides and low density lipoproteins (LDL) [41].

In addition, Green tea consumption by diabetic individuals has been shown to be beneficial. Indeed, ingestion of $582 \mathrm{mg} /$ day for 12 days of green tea catechins significantly reduced waistline and visceral fat. Further, consumption of green tea polyphenols significantly increased adiponectin levels. Furthermore, green tea polyphenols improved insulin production as demonstrated by reduced haemoglobin A1c levels [40].

Although there has been evidence to suggest adiponectin as an anti-obesity adipocytokine, there has also been conflicting evidence that indicates green tea is ineffective in increasing adiponectin levels [43]. A study by Basu et al. [45] involved human subjects who were suffering from obesity and metabolic syndrome. Following 8 weeks of consumption of decaffeinated green tea (4 cups per day, equivalent to $400 \mathrm{mg}$ of EGCG per day) or decaffeinated green tea extract capsule ( 2 capsules per day with 4 cups of water, equivalent to $460 \mathrm{mg}$ of EGCG per day) did not significantly alter adiponectin levels. A similar findings were reported by Ryu et al [46] in a study involving type 2 diabetic human subjects who consumed green tea (9 grams green tea per $900 \mathrm{mls}$ water) for a 4 week.

Although there is more evidence that demonstrates increase adiponectin levels following green consumption and this potential of green tea can be used to combat obesity, there are reports that suggest green tea consumption does not alter adiponectin levels. Therefore, further studies are warranted to fully explore the potential effect of green tea on adiponectin and other pathways that play a role in controlling fat production and metabolism. One potential target is insulin sensitivity. Indeed, several studies suggested that adiponectin plays a larger role in insulin sensitivity in obese individuals with type 2 diabetes, rather than effecting physiology in healthy individuals or in weight loss [47]. 
Furthermore, a recent study by Nirengi et al [48] demonstrating increase in brown adipose tissue in healthy women post consumption of a beverage containing green tea catechins suggesting a possible mechanism for the increase in energy expenditure.

\section{CONCLUSION}

Tea consumption is a common practice in many cultures and communities around the world. There are evidence that suggest green tea has been used a medicinal remedy for many conditions. Green tea consumption has been shown to be associated with multiple beneficial biological outcomes. One of these beneficial properties of green tea is reduction in body weight via the modulation of leptin and adiponectin. There are many reports that demonstrate decreased leptin levels following green tea consumption. Leptin directly affects body weight by increasing energy expenditure and fat oxidation. Furthermore, it has been shown that adiponectin levels are increased post green tea ingestion. Adiponectin has been shown to play a role in weight loss indirectly through altering insulin sensitivity. In order to utilise green tea as an anti-obesity treatment further research needs to be conducted to fully understand the mechanisms by which green tea functions to decrease weight, and therefore to decrease the main risk factor of CVD, obesity.

\section{CONFLICT OF INTEREST}

The authors confirm that this article content has no conflict of interest.

\section{ACKNOWLEDGEMENTS}

None

\section{REFERENCES}

[1] Sumpio BE, Cordova AC, Berke-Schlessel DW, Qin F, Chen QH. Green tea, the "Asian paradox," and cardiovascular disease. Journal of the American College of Surgeons. 2006;202(5):813-25.

[2] Heiss ML, Heiss RJ. The story of tea: a cultural history and drinking guide: Random House Digital, Inc.; 2007.

[3] Harbowy ME, Balentine DA, Davies AP, Cai Y. Tea chemistry. Critical reviews in plant sciences. 1997;16(5):415-80.

[4] Chacko SM, Thambi PT, Kuttan R, Nishigaki I. Beneficial effects of green tea: a literature review. Chinese medicine. 2010;5(1):1.

[5] Babu A, Pon V, Liu D. Green tea catechins and cardiovascular health: an update. Current medicinal chemistry. 2008;15(18):1840-50.

[6] AIHW. What is cardiovascular disease? 2016 [cited 2016 1.10]. Available from: http://www.aihw.gov.au/cardiovascular-disease/what-is-cvd/. [7] Wolfram S, Wang Y, Thielecke F. Anti-obesity effects of green tea: From bedside to bench. Molecular nutrition \& food research. 2006;50(2):176-87.

[8] Serisier S, Leray V, Poudroux W, Magot T, Ouguerram K, Nguyen P. Effects of green tea on insulin sensitivity, lipid profile and expression of PPAR $\alpha$ and
PPAR $\gamma$ and their target genes in obese dogs. British Journal of Nutrition. 2008;99(06):1208-16.

[9] Mosawy S, Gaiz A, Karaksha A, Singh I. The Green Tea Extract Epigallocatechin Gallate Inhibits Human Platelet Function but not Plasma Coagulation. International Journal of Prevention and Treatment. 2016;5(2):17-21.

[10] Kadowaki T, Yamauchi T, Kubota N, Hara K, Ueki $\mathrm{K}$, Tobe K. Adiponectin and adiponectin receptors in insulin resistance, diabetes, and the metabolic syndrome. The Journal of clinical investigation. 2006;116(7):1784-92.

[11] Ricci R, Bevilacqua F. The potential role of leptin and adiponectin in obesity: a comparative review. The Veterinary Journal. 2012;191(3):292-8.

[12] Gautron L, Elmquist JK. Sixteen years and counting: an update on leptin in energy balance. The Journal of clinical investigation. 2011;121(6):2087-93.

[13] Brennan AM, Mantzoros CS. Drug Insight: the role of leptin in human physiology and pathophysiologyemerging clinical applications. Nature Reviews Endocrinology. 2006;2(6):318-27.

[14] Lavie CJ, Milani RV, Ventura HO. Obesity and cardiovascular disease: risk factor, paradox, and impact of weight loss. Journal of the American College of Cardiology. 2009;53(21):1925-32.

[15] Hossain P, Kawar B, El Nahas M. Obesity and diabetes in the developing world-a growing challenge. New England journal of medicine. 2007;356(3):213-5.

[16] Dixon JB. The effect of obesity on health outcomes. Molecular and cellular endocrinology. 2010;316(2):104-8.

[17] WHO. Top 10 Causes of Death 2012 [cited 2016 01.10]. Available from: http://www.who.int/mediacentre/factsheets/fs310/en/index2. html.

[18] Poirier P, Giles TD, Bray GA, Hong Y, Stern JS, PiSunyer FX, et al. Obesity and cardiovascular disease pathophysiology, evaluation, and effect of weight loss. Arteriosclerosis, thrombosis, and vascular biology. 2006;26(5):968-76.

[19] Nakajima K, Nakano T, Tanaka A. The oxidative modification hypothesis of atherosclerosis: the comparison of atherogenic effects on oxidized LDL and remnant lipoproteins in plasma. Clinica Chimica Acta. 2006;367(1):36-47.

[20] Tedgui A, Mallat Z. Cytokines in atherosclerosis: pathogenic and regulatory pathways. Physiological reviews. 2006;86(2):515-81.

[21] Reilly MP, Li M, He J, Ferguson JF, Stylianou IM, Mehta NN, et al. Identification of ADAMTS7 as a novel locus for coronary atherosclerosis and association of $\mathrm{ABO}$ with myocardial infarction in the presence of coronary atherosclerosis: two genome-wide association studies. The Lancet. 2011;377(9763):383-92.

[22] Cabrera C, Artacho R, Giménez R. Beneficial effects of green tea- a review. Journal of the American College of Nutrition. 2006;25(2):79-99.

[23] Nigro E, Scudiero O, Monaco ML, Palmieri A, Mazzarella G, Costagliola C, et al. New insight into adiponectin role in obesity and obesity-related diseases. BioMed research international. 2014;2014. 
[24] Banks WA, Coon AB, Robinson SM, Moinuddin A, Shultz JM, Nakaoke R, et al. Triglycerides induce leptin resistance at the blood-brain barrier. Diabetes. 2004;53(5):1253-60.

[25] Antoniades C, Antonopoulos A, Tousoulis D, Stefanadis C. Adiponectin: from obesity to cardiovascular disease. obesity reviews. 2009;10(3):269-79.

[26] Tilg H, Moschen AR. Adipocytokines: mediators linking adipose tissue, inflammation and immunity. Nature Reviews Immunology. 2006;6(10):772-83.

[27] Wang M, Crisostomo PR, Herring C, Meldrum KK, Meldrum DR. Human progenitor cells from bone marrow or adipose tissue produce VEGF, HGF, and IGF-I in response to TNF by a p38 MAPK-dependent mechanism. American Journal of Physiology-Regulatory, Integrative and Comparative Physiology. 2006;291(4):R880-R4.

[28] Achike FI, To NHP, Wang H, Kwan CY. Obesity, metabolic syndrome, adipocytes and vascular function: a holistic viewpoint. Clinical and Experimental Pharmacology and Physiology. 2011;38(1):1-10.

[29] Porth C, Matfin G. Pathophysiology: concepts of altered health states. 8 ed: Lippincott Williams \& Wilkins; 2009.

[30] Smith C, Mocanu M, Davidson S, Wynne A, Simpkin J, Yellon D. Leptin, the obesity-associated hormone, exhibits direct cardioprotective effects. British journal of pharmacology. 2006;149(1):5-13.

[31] Feldt-Rasmussen U. Thyroid and leptin. Thyroid. 2007;17(5):413-9.

[32] Frühbeck G. Intracellular signalling pathways activated by leptin. Biochemical Journal. 2006;393(1):7-20.

[33] Zheng H, Berthoud H-R. Neural systems controlling the drive to eat: mind versus metabolism. Physiology. 2008;23(2):75-83.

[34] ZHENG G, SAYAMA K, OKUBO T, JUNEJA LR, OGUNI I. Anti-obesity effects of three major components of green tea, catechins, caffeine and theanine, in mice. In Vivo. 2004;18(1):55-62.

[35] Sayama K, Lin S, Zheng G, Oguni I. Effects of green tea on growth, food utilization and lipid metabolism in mice. In vivo (Athens, Greece). 1999;14(4):481-4.

[36] Auvichayapat P, Prapochanung M, Tunkamnerdthai O, Sripanidkulchai B-o, Auvichayapat N, Thinkhamrop B, et al. Effectiveness of green tea on weight reduction in obese Thais: A randomized, controlled trial. Physiology \& behavior. 2008;93(3):486-91.

[37] Lee M-S, Kim C-T, Kim Y. Green tea (-)epigallocatechin-3-gallate reduces body weight with regulation of multiple genes expression in adipose tissue of diet-induced obese mice. Annals of Nutrition and Metabolism. 2009;54(2):151-7.

[38] Dulloo AG, Duret C, Rohrer D, Girardier L, Mensi $\mathrm{N}$, Fathi M, et al. Efficacy of a green tea extract rich in catechin polyphenols and caffeine in increasing 24-h energy expenditure and fat oxidation in humans. The American journal of clinical nutrition. 1999;70(6):1040-5.

[39] Habib CN, Al-Abd AM, Tolba MF, Khalifa AE, Khedr A, Mosli HA, et al. Leptin influences estrogen metabolism and accelerates prostate cell proliferation. Life sciences. 2015;121:10-5.

[40] Lorenz M, Paul F, Moobed M, Baumann G, Zimmermann BF, Stangl K, et al. The activity of catechol-Omethyltransferase (COMT) is not impaired by high doses of epigallocatechin-3-gallate (EGCG) in vivo. European journal of pharmacology. 2014;740:645-51.

[41] Myers MG, Cowley MA, Münzberg H. Mechanisms of leptin action and leptin resistance. Annu Rev Physiol. 2008;70:537-56.

[42] Chandran M, Phillips SA, Ciaraldi T, Henry RR. Adiponectin: more than just another fat cell hormone? Diabetes care. 2003;26(8):2442-50.

[43] Imatoh T, Tanihara S, Miyazaki M, Momose Y, Uryu $\mathrm{Y}$, Une $\mathrm{H}$. Coffee consumption but not green tea consumption is associated with adiponectin levels in Japanese males. European journal of nutrition. 2011;50(4):279-84.

[44] Tian C, Ye X, Zhang R, Long J, Ren W, Ding S, et al. Green tea polyphenols reduced fat deposits in high fat-fed rats via erk1/2-PPAR $\gamma$-adiponectin pathway. PloS one. 2013;8(1):e53796.

[45] Basu A, Du M, Sanchez K, Leyva MJ, Betts NM, Blevins $S$, et al. Green tea minimally affects biomarkers of inflammation in obese subjects with metabolic syndrome. Nutrition. 2011;27(2):206-13.

[46] Ryu O, Lee J, Lee K, Kim H, Seo J, Kim S, et al. Effects of green tea consumption on inflammation, insulin resistance and pulse wave velocity in type 2 diabetes patients. Diabetes research and clinical practice. 2006;71(3):356-8.

[47] McMurry J, Castellion M, Ballantine D, Hoeger C, Peterson V. Fundamentals of general, organic, and biological chemistry. 2010.

[48] Nirengi S, Amagasa S, Homma T, Yoneshiro T, Matsumiya S, Kurosawa $\mathrm{Y}$, et al. Daily ingestion of catechin-rich beverage increases brown adipose tissue density and decreases extramyocellular lipids in healthy young women. SpringerPlus. 2016;5(1):1363. 